Evan Greenberg ${ }^{1 \text { [0000-0001-5055-6726] }}$, Anna Mavrogianni ${ }^{2 \text { [0000-0002-5104-1238] }}$ and Sean Hanna ${ }^{3}[0000-0002-0529-2116]$

\title{
Toward a Spatial Model for Outdoor Thermal Comfort
}

\author{
${ }^{1}$ Bartlett School of Architecture, UCL, 22 Gordon Street, London WC1H 0QB UK \\ evan.greenberg@ucl.ac.uk \\ ${ }^{2}$ Bartlett School of Architecture, UCL, 22 Gordon Street, London WC1H 0QB UK \\ s.hanna@ucl.ac.uk \\ ${ }^{3}$ UCL IEDE, Central House, 14 Upper Woburn Place, London WC1H 0NN UK \\ a.mavrogianni@ucl.ac.uk
}

\begin{abstract}
This paper presents the methods used and initial data analysis implemented toward the development of a spatial-architectural model for outdoor thermal comfort. Variables have been identified from existing thermal comfort models and qualified relative to their spatiality while data from a subset of these has been collected for preliminary statistical modelling, used to develop an initial understanding of the significance of spatial variables on thermal comfort. Although the specific analyses undertaken thus far are only introductory and do not provide definitive conclusions on their significance, the current study begins to bring to light the possibility that some explicitly spatial variables may explain enough variation in outdoor thermal comfort to justify the creation of a thermal comfort model for architectural design. The benefits of this approach on urban design and urban analysis is discussed.
\end{abstract}

Keywords: Thermal Comfort, Spatial Analysis, Regression Modelling.

\section{$1 \quad$ Introduction}

It has been suggested that microclimatic variation in the urban environment can promote the increased use and creation of programmed space which contributes to the social and cultural life of the city [1] while increasing the thermal comfort range [2]. However, determining comfort in a dynamic urban environment is not a trivial task. Over the past 100 years or so, hundreds of indices have been developed in order to arrive at the calculation of a single value of thermal comfort, focusing primarily on the six basic human thermal comfort parameters. From initial field studies using a kata thermometer [3] undertaken in the early part of the $20^{\text {th }}$ century to the most contemporary computationally-driven indices, such as the Universal Thermal Climate Index [4], this quantification of thermal comfort is largely driven through the understanding of the environment, human physiology and behavior. Both the Modified Temperature Index [5] and the Actual Sensation Vote [6] address the relationship between a model of thermal comfort and space - and specifically, the outdoor urban environment - but ultimately consider these same environmental physiological and 
behavioral parameters which cannot necessarily be controlled through architectural design. If architects can only design space, rather than the climate of a space or the physiology of a human within a space, it is crucial to develop an understanding of the spatial variables affecting thermal comfort. This paper presents the methods used and initial data analysis implemented toward the development of a spatial-architectural model for outdoor thermal comfort and discusses the implications of such a model on architectural design. The methods and preliminary analysis presented in this paper are part of a larger research investigating the extent of significance of these spatial variables on thermal comfort.

\section{Methods}

\subsection{Identification of Parameters and Parameter Classes}

While this research ultimately aims to propose a model for outdoor thermal comfort, a review of 64 indoor and outdoor thermal comfort indices has been conducted in order to gain a broad view of the variables included within the models and to identify those which are spatial in character. Previous cataloguing of thermal comfort indices undertaken by de Freitas and Grigorieva [7], Blazejczyk, Epstein, et al. [8], Auliciems and Szokolay [9] and Parsons [10] were used as primary sources of identification.

In order to categorize variables considered in each index, six variable classes have been identified as physiological, behavioral, environmental, psychological, positional and demographical. Physiological variables are those that relate to the body functions of the individual human body. Behavioral variables are those actions which are carried out by the individual. Environmental variables are associated with the physical conditions and surroundings. Psychological variables ${ }^{1}$ are those that are personal, subjective or related to history, memory or thought processes of the individual. Positional variables concern the location or climatic context at which the index was calculated or aimed. Finally, demographical variables belong to those characteristics of a specific population type for which the index was calculated or aimed.

\subsection{Data Recording}

The study of variables within the thermal comfort indices explored led to the identification of 96 distinct variables. In order to determine the significance of the variables on thermal comfort, an analysis of all independent variables was required. Therefore, literature on each of the 64 thermal comfort indices identified has been reviewed fully

\footnotetext{
${ }^{1}$ Psychological variables included here, aside from the variable decision, are based on the definitions put forward by Nikolopoulou and Steemers [11]. Naturalness is defined as the existence of naturally-produced conditions; time is defined as the length of time exposed to conditions in a space; environmental stimulation is considered as the presence of variable climatic conditions; perceived control is the perceived ability to change location; short-term experience is the memory or history of exposure to immediately previous conditions; decision is considered as the determined selection of a specific environment, as defined by the authors.
} 
in order to appropriate values for each variable across all indices. A number of assumptions have been made within this method in order to reduce the number of independent variables. For instance, any indoor environment is assumed to be without direct solar radiation. A school environment, for example, will likely have students sitting at their desks which would be abstracted through an appropriate metabolic rate. In this regard, it would therefore be acceptable to eliminate an occupational variable. Similarly, the individual country which has been identified as a specific variable has been omitted; this information can be accounted for through psychological parameters, due to the fact that the overarching cultural significance to thermal comfort is acclimatization, and acclimatization can be considered within variables such as time (time spent within a space) and long-term experience (experience built over numerous visits to a space or over long periods of time). Furthermore, in an attempt to include as many data points as possible within the global set, assumptions have been made for those data points that were missing based on logical deduction. For example, where air humidity or air pressure values have been omitted within a data set in the literature, a logical assumption has been made using published weather data sets (TMY3 or IWEC, for example) for those specific geographical locations in order to arrive at an approximate value for these variables.

After recording data collected during original field experiments described in the literature and logically assumed, the matrix of indices and variables was edited in order to arrive at a data set with a large portion of recorded data points, resulting in a set of 753 data points across 27 variables and 14 indices.

\subsection{Preliminary Analysis of Thermal Comfort Data}

It is beneficial to perform preliminary analysis on the data set, in order to develop an understanding of patterns and relationships between each independent variable and the dependent variable. In doing so, a scatterplot was generated for each of the 24 continuous independent variables related to thermal comfort. While three ordinal variables have been identified, they have been omitted from this study at present due to the complexity in their analysis, but will be included in future studies. The Pearson's Correlation Coefficient, or $\mathrm{r}$, was calculated for each independent variable relative to the dependent variable, and ranked [Figure 1] for the purposes of comparison. Due to the large variation in data sets from different thermal comfort indices, a specific judgement on how to interpret the correlation coefficient has not been made. However, it can be noted that five variables have a correlation coefficient above \pm 0.6 , four variables between \pm 0.4 and \pm 0.59 , while the remaining 15 variables showed low to no correlation.

\subsection{Single Variable Linear Regression and Best-Fit Curves}

Further to calculating the Pearson's Correlation Coefficient for each continuous independent variable and thermal comfort, the coefficient of determination or $\mathrm{R}^{2}$, was calculated, and the best-fit curve and its equation was found for those variables with $\mathrm{R}^{2}$ values greater than 0.50 [Figure 2]. While $\mathrm{r}$ gives the correlation and positive- 
negative relationship between an independent and its dependent variable, $\mathrm{R}^{2}$ returns the percentage of data that can be explained by the best-fit curve [12].

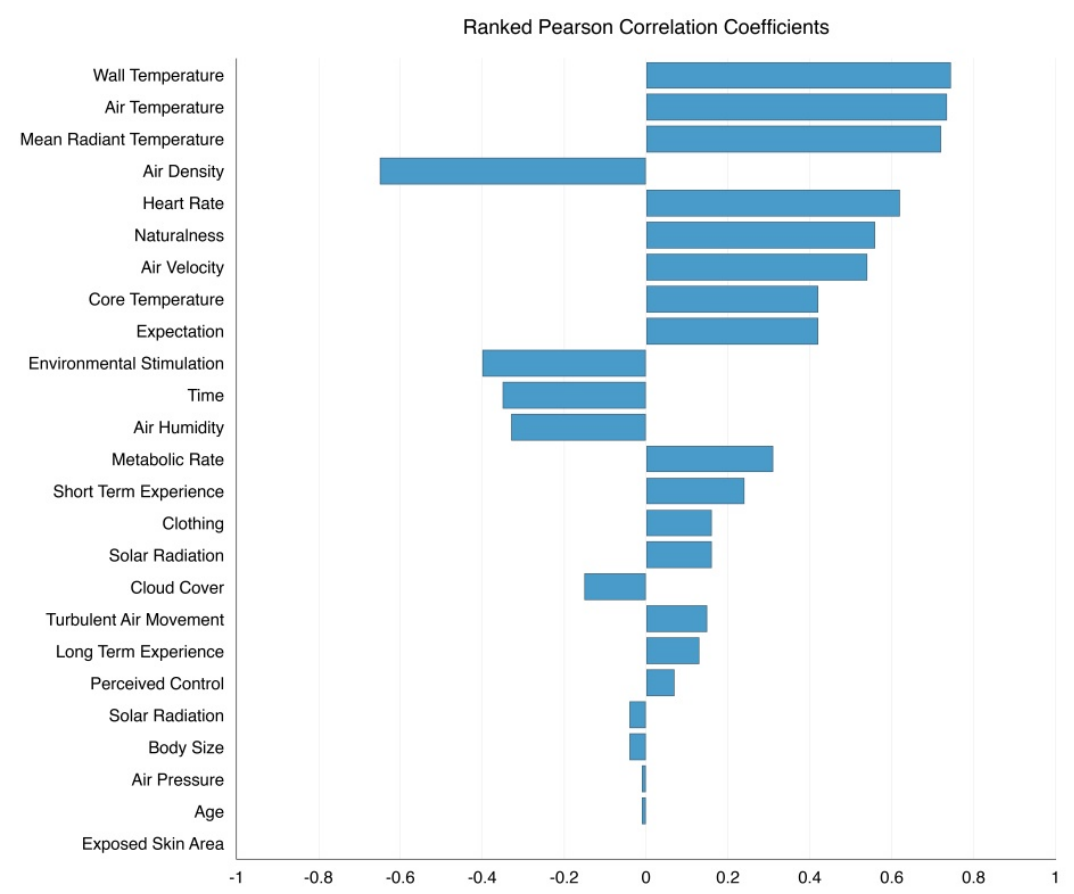

Fig. 1. Ranked Pearson correlation coefficients of independent variables effecting thermal comfort. Image courtesy of authors.

Only three variables (wall temperature, air temperature and mean radiant temperature) have $\mathrm{R}^{2}$ values over 0.5 , meaning that approximately $50 \%$ of the variation in the predicted thermal comfort levels can be explained by the variation in one of these independent variables alone. Four independent variables have $\mathrm{R}^{2}$ values between 0.2 and 0.5 , and five additional variables have $\mathrm{R}^{2}$ values between 0.1 and 0.2 . Twelve remaining variables have $\mathrm{R}^{2}$ values below 0.1 . All continuous independent variables have been ranked [Figure 3] for the purposes of comparison.

There is no doubt that the above quantitative analysis holds great value; at the same time, however, it is important to view the data qualitatively in order to discern patterns and potential non-linear relationships that may not accurately explain the data $[13,14]$. For this reason, each scatter plot has been assessed visually. For a number of scatterplots, it has been found that specific indices caused outliers to change the coefficient of determination and the resulting best-fit curve. In these instances, data from these indices have been removed and the coefficient of determination recalculated. An altered ranking of continuous independent variables following this visual analysis has been developed [Figure 4] for the purposes of comparison. 


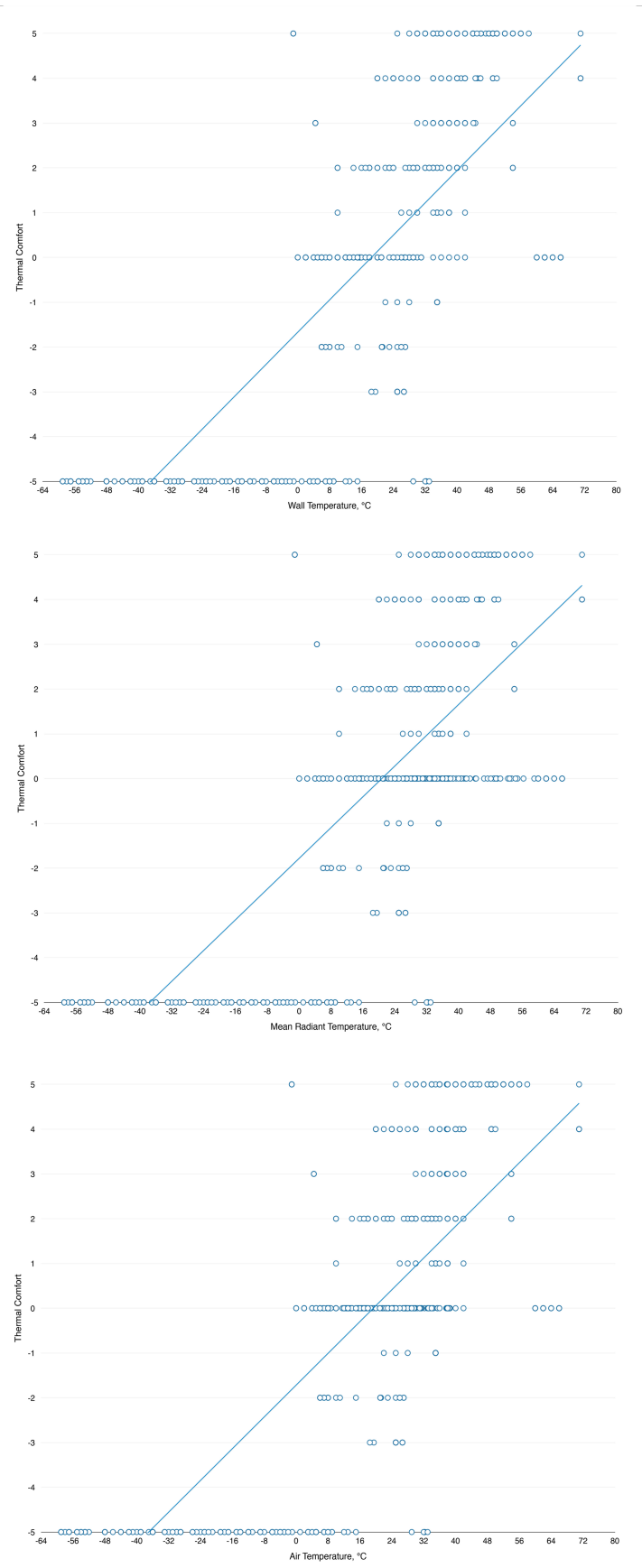

Fig. 2. Scatter plots for three continuous independent variables plotted against thermal comfort, with best-fit curves with $\mathrm{R}^{2}$ values greater than 0.50 . Image courtesy of authors. 


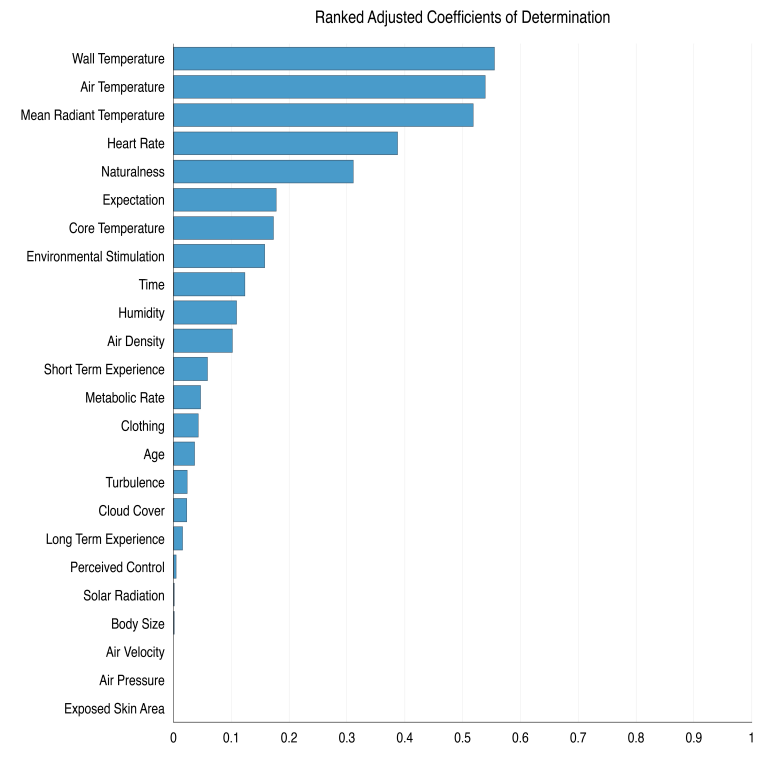

Fig. 3. Ranked coefficients of determination of independent variables effecting thermal comfort. Image courtesy of authors.

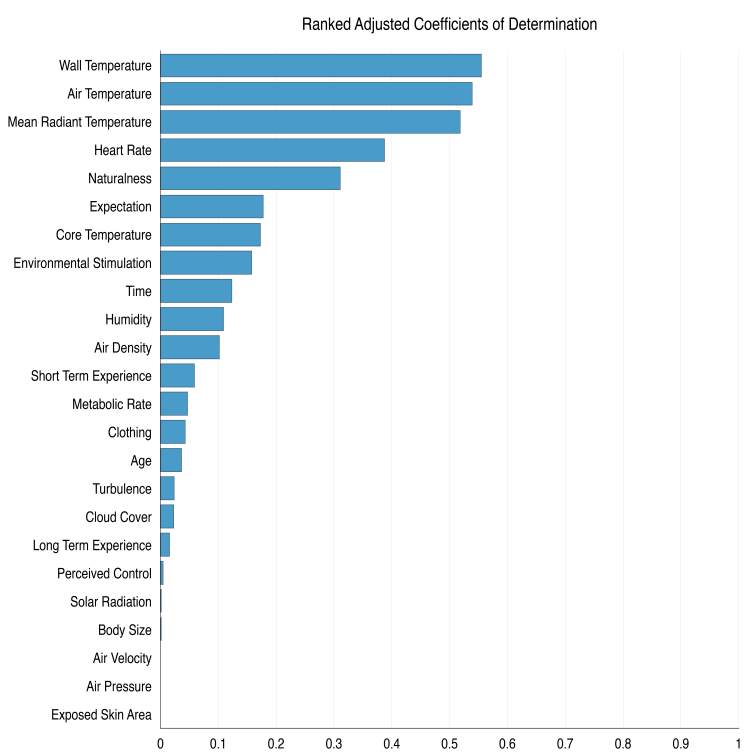

Fig. 4. Ranked adjusted coefficients of determination of independent variables effecting thermal comfort. Image courtesy of authors. 


\subsection{Analysis of Parameter Spatiality}

In making the distinction between those parameters that are spatial from those that are not, parameters that are at a scale larger than the space under examination, such as weather patterns, as well as those that are controlled by or linked to the individual have not been included. Because all indices identified do not take into account the same parameters, it is not possible to compare them through absolute terms. There fore, a system of relative spatiality [Figure 5], as defined by orders of magnitude, has been defined in order to determine those parameters which are to be included in a proposed spatial model for thermal comfort. Those parameters which are inherently spatial are considered of the zeroth order; those which are directly affected by space are of the first order; those which are affected by first-order parameters are of the second order, and so on. Those parameters which are in no way affected by space are of an infinite order. Including all parameters which are in some way spatial (while omitting those which are of an infinite order) would result in a highly complicated model with numerous variables which could not be controlled architecturally. Therefore only those that are of a zeroth or first order will be considered as spatial within a new model for thermal comfort as they provide a real opportunity for spatial impact in the design process. It is important to mention that those parameters which have been identified previously as discreet but are in fact specific instances of more generic parameters have been considered only in the generic. For example, metabolic rate, or activity, has been identified in various specific quantities such as light activities or moderate exercise, but in evaluating their spatiality are considered together as metabolic rate.

Determining the order of spatiality of all parameters results in the establishment of 14 spatial parameters whose significance toward thermal comfort can be considered. These parameters include: location (indoors or outdoors), naturalness, time, environmental stimulation, perceived control, short-term experience, decision, mean radiant temperature, wall temperature, solar radiation, air velocity, turbulent air movement, and metabolic rate of three parameter classes: environment, psychology, and behavior.

\section{Discussion}

Understanding the relationships between all independent variables and their effect on thermal comfort is crucial in developing a new model for thermal comfort. However, it is paramount to understand the particular significance of those variables that are spatial so that they may be leveraged by architects in the design of architectural space. The above statistical analysis has shown that the regression equation for the highest significance of an independent variable (wall temperature) can account for only approximately $50 \%$ of all variation in the dependent variable (thermal comfort).

Although this may at first glance appear to be a very low significance, this is difficult to ascertain from this study alone. The collected data set includes data points from different models and therefore likely possesses hierarchical relationships that should be statistically considered in future. 


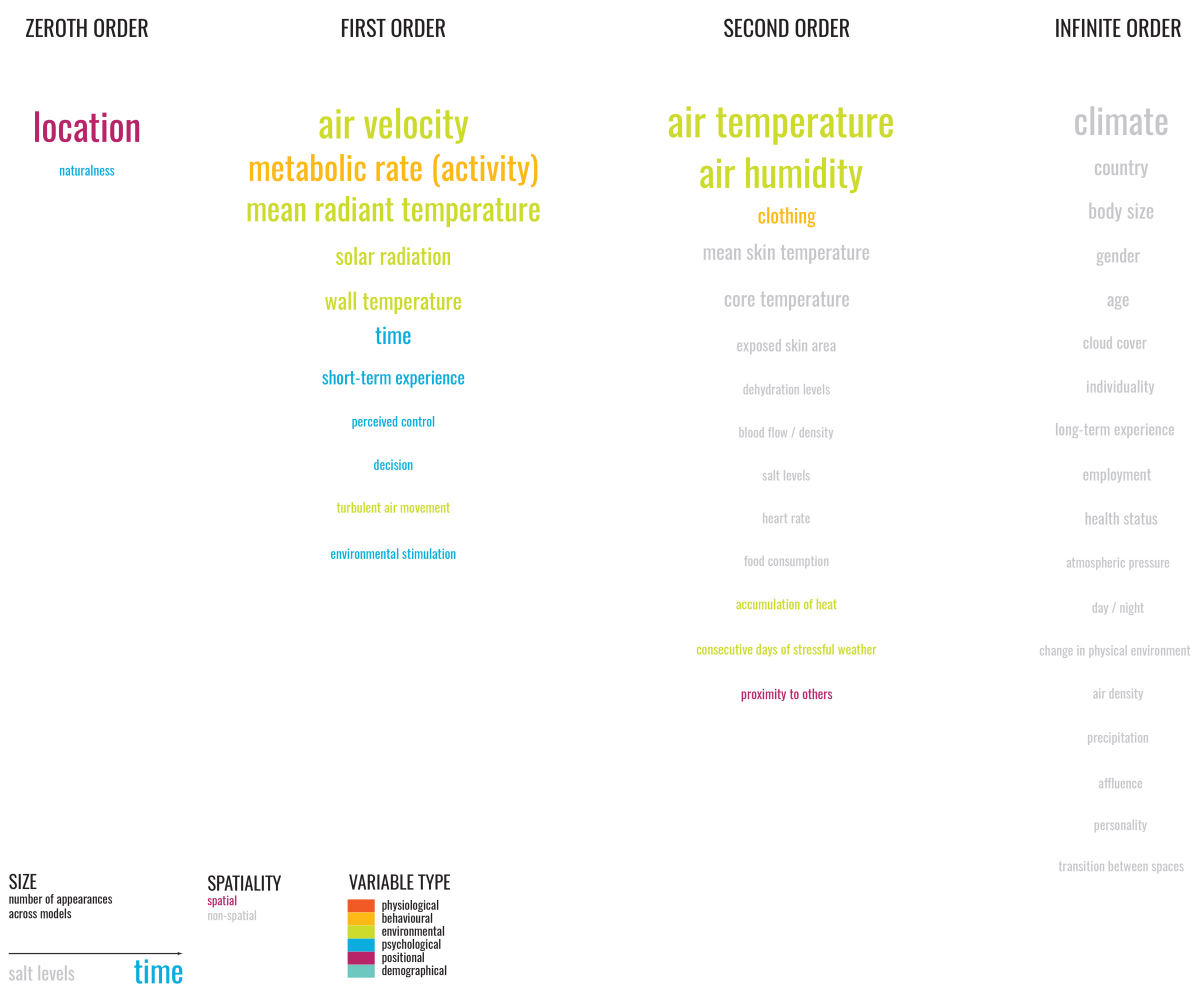

Fig. 5. Qualitative analysis of variable spatial order, outlining variable type, its relative occurrence across thermal comfort indices and its spatial order. Image courtesy of authors.

While it is beneficial to approach each individual independent variable analytically, it is clear that thermal comfort cannot be explained by one independent variable alone, and the relationship between independent variables may better explain their significance on thermal comfort. A regression model will be developed in the next phase of this research in order to determine the independent variables which affect thermal comfort, and distinctly, the significance of those independent variables that are spatial. Particular emphasis will be made on exploring hierarchical statistical models in order to account for the numerous existing indices studied and the varied data that has been recorded.

This presents a noteworthy and consequential opportunity for designers to modify the conventional approach to design, where an independent variable, such as time or naturalness, for example, may hold similar or greater importance than traditional design variables such as morphology or orientation. While a definitive conclusion cannot be made from the specific analyses undertaken thus far, the current study begins to bring to light the possibility that some explicitly spatial variables may explain enough variation in outdoor thermal comfort to justify the creation of a thermal comfort model for architectural design. 


\section{Future Outlook}

\subsection{Thermal Comfort as an Urban Design Tool}

This new spatial-architectural model for thermal comfort has the potential to be used as an urban design tool, capable of aiding in the design of outdoor spaces, their adjacencies and ultimately their programs. This presents a significant shift from current thermal comfort models, often used as verification tools or as a mechanism to inform users of how behavior, such as the increase or decrease of clothing layers, must be amended. In contrast, if it is assumed that spatial parameters such as wall and mean radiant temperatures as well as naturalness, for example, are statistically significant in determining thermal comfort, this provides architects an opportunity to leverage design decisions around these parameters. For example, the materiality and color of external surfaces of buildings and ground may hold particular importance, as the surface albedo would contribute directly to both wall temperature and mean radiant temperature. The materiality of a building system has a direct relationship to structure; in this case, it is not difficult to imagine that structural systems, intrinsically tied to architectural quality and morphology, could thus begin to connect closely to outdoor thermal comfort. Designers too could begin to develop novel approaches to solar access or wind flow, for instance, so that artificial lighting or ventilation is decreased and naturalness is amplified.

\subsection{Thermal Comfort as an Urban Analysis Tool}

Furthermore, this thermal comfort model can be compared to existing spatial analysis methods to develop an understanding of the ability for thermal comfort to predict spatial use. While the Space Syntax method, for example, employs topological and geometric analyses to determine spatial use [15], the thermal comfort model sought in this research provides an opportunity to explore spatial analysis through architectural design. This alteration to an approach toward urban analysis may allow for an enhancement in empathic design in a field where increased quantitative design is often seen as lacking in human quality.

\section{Conclusions}

The research presented here demonstrates the methods and preliminary statistical analysis of a set of independent variables in determining thermal comfort. Pearson correlation coefficient values have been calculated and ranked in order to develop an understanding of the relative correlations of independent variables and thermal comfort. Coefficients of determination have also been calculated; while the greatest significance has only shown a 50\% description for output data, a high proportion of independent variables have $\mathrm{R}^{2}$ values well above the mean value; these findings begin to suggest that the relative significance of independent variables may hold importance, rather than specific absolute values. Only a multilevel regression analysis of 
all independent variables will allow for a real understanding of the significance of independent variables on thermal comfort. Nonetheless, a new approach to design may emerge from the utilization of a spatial model for thermal comfort, where design parameters that affect those variables have great importance and potentially begin to transform analysis-driven architectural design toward a humanistic perspective.

\section{References}

1. Greenberg, E. \& Jeronimidis, G. Variation and Distribution: Forest Patterns as a Model for Urban Morphologies. Architectural Design 83(4). London: Wiley (2013).

2. Steemers, K., Ramos, M., Sinou, M. Urban Diversity. In: Steemers, K., Steane, M. A. (Eds.) Environmental Diversity in Architecture. Abingdon: Spon Press (2004).

3. Hill, L., Griffith, O., Flack, M. The Measurement of the Rate of Heat-Loss at Body Temperature by Convection, Radiation and Evaporation, Philosophical Transactions of the Royal Society of London, Series B, Containing Papers of a Biological Character, 207, 183220 (1916).

4. Jendritzky, G., Maarouf, A., Staiger, H. Looking for a Universal Thermal Climate Index (UTCI) for Outdoor Applications. Proceedings of the Windsor Conference on Thermal Standards, 5-8 (2001).

5. Adamenko, V., Khairullin, K. Evaluation of Conditions under Which Unprotected Parts of the Human Body May Freeze in Urban Air During Winter. Boundary-Layer Meteorology, 2, 510-518 (1972).

6. Nikolopoulou, M. Designing Open Spaces in the Urban Environment: A Bioclimatic Approach, Centre for Renewable Energy Sources, EESD, FP5 (2004).

7. De Freitas, C. R., Grigorieva, E. A. A Comprehensive Catalogue and Classification of Human Thermal Climate Indices. International Journal of Biometeorology, 59, 109-20 (2015).

8. Blazejczyk, K., Epstein, Y., Jendritzky, G., Staiger, H., Tinz, B. Comparison of UTCI to Selected Thermal Indices. International Journal of Biometeorology, 56, 515-35 (2012).

9. Auliciems, A., Szokolay, S. V. Thermal Comfort. PLEA (1997).

10. Parsons, K. Human Thermal Environments: The Effects of Hot, Moderate, and Cold Environments on Human Health, Comfort, and Performance, CRC Press (2014).

11. Nikolopoulou, M., Steemers, K. Thermal Comfort and Psychological Adaptation as a Guide for Designing Urban Spaces. Energy and Buildings, 35, 95-101 (2003).

12. Moore, D. S., McCabe, G. P. Introduction to the Practice of Statistics, New York, W. H. Freeman And Company (2006).

13. Anscombe, F. J. Graphs In Statistical Analysis. The American Statistician, 27, 17-21 (1973).

14. Matejka, J. \& Fitzmaurice, G. Same Stats, Different Graphs: Generating Datasets with Varied Appearance and Identical Statistics through Simulated Annealing. Proceedings of the 2017 Chi Conference on Human Factors in Computing Systems. Denver, Colorado, USA: ACM (2017).

15. Al Sayed, K., Turner, A., Hillier, B., Iida, S., Penn, A. Space Syntax Methodology, London, Bartlett School Of Architecture, UCL (2014). 